\title{
Adsorption of lomustin anticancer drug on the surface of carbon nanotube: A theoretical study
}

\author{
aDepartment of Inorganic Chemistry, Faculty of \\ Chemistry, Tehran North Branch, Islamic Azad \\ University, Tehran, Iran \\ ${ }^{b}$ Department of Chemistry, Yadegar-e-Imam \\ Khomeini (RAH) Shahre-rey Branch, Islamic Azad \\ University, Tehran, Iran
}

Maryam Afshar $^{\mathrm{a}}$ |Roya Ranjineh khojasteha ${ }^{\text {|Roya Ahmadib,* }}$
The present study aimed to assess the adsorption of Lomustin on the single-walled carbon nanotube which has been examined using Density Functional Theory (DFT), agent in a solvent phase (water) at the B3LYP/6-31G (d) theoretical level. Initially, the structures of Lomustin, carbon nanotube, and Lomustin complexes with carbon nanotubes were designed in Gauss View in three different conformers and were optimized geometrically, on which IR and frontier molecular orbital computations were carried out. Adsorption energy values, Gibbs free energy changes $\left(\Delta \mathrm{G}_{\mathrm{ad}}\right)$, adsorption enthalpy changes $\left(\Delta \mathrm{H}_{\mathrm{ad}}\right)$, and equilibrium thermodynamic constants were estimated. The results showed that adsorption process was spontaneous, exothermic and nonequilibrium. The values of specific heat capacity and adsorption enthalpy indicate that this nanostructure can be used to build new thermal sensors to measure Lomustin. The results of molecule orbitals estimations showed that energy gap, after drug absorption on the nanotube surface, decreased significantly and the values of chemical hardness and dipole moment were studied after the interaction of drug with adsorbent and the results showed that drug solubility and reactivity, after adsorption on carbon nanotubes, increased significantly. According to the obtained results for adsorption of Lomustin, this nanostructure can be used as a sensing material in building new electrochemical sensors to measure this drug.

\section{KEYWORDS}

Lomustin; drug delivery; detection; density functional theory; single-walled carbon nanotube.

\section{Introduction}

Carbon nanotubes discovery in 1991 led to extensive studies on carbon nanotubes and their applications. The main reasons were their structural evolution, small size, low density, high hardness, high strength, and excellent electrical properties. As a result, carbon nanotubes can be used extensively in reinforcement of materials, chemical sensors, drug delivery, and electronic science. On the other hand, in recent years, the application of carbon nanotubes as drug carriers and biological molecules has been taken into consideration in medicine and drug delivery. Carbon nanotubes, with unique properties such as high surface to volume ratio and high chemical stability, have high capacity and ability to absorb chemicals. Monolayer carbon nanotubes are significantly hard and strong conductors of electricity and conduct heat and for these properties, they are used in electronic industry. Also, carbon nanotubes, due to high biological compatibility and strength, have high potential for biomedical applications. Therefore, in medicine, they can be used in controlled and purposeful drug delivery. They are delivered through different 
routes such as oral, injection, and other methods and, for this reason, in addition to influencing the whole body, the side effects occur and to achieve a certain effect, high amounts of the drug should be consumed [14]. Now, the mixture of drugs and some nanotubes is very important to create anticancer drugs against tumours and their destruction. Therefore, only tumours are attacked and other textures do not suffer from side effects (such as hair loss after chemotherapy) [4-5]. One of the properties of nanotubes is acidic environment while other body cells indicate $\mathrm{pH}=7$ (neutral). They are called objective cells because they direct anticancer drug toward cancerous masses with the help of nanotubes. Carbon nanotubes are ideal materials for different applications in this context [6-7]. These properties include potential biological compatibility in drug delivery systems as well as their excellent role as drug carriers with high selective delivery and sensitivity. Moreover, carbon nanotubes as multifunctional carriers, are suggested and designed for drug delivery that their multifunctional physical and chemical properties provide covalent and non-covalent bonds of several drugs and create a rational structure for drug development based on nanoscale [8-9]. Various cell absorption pathways of nanotubes are highly dependent on the type of nanotubes and biological molecules on their surface. Functional nanotubes related to molecules with low weight (with a covalent bond) seem to penetrate into the cell plasma membrane through energy-independent mechanisms and their passage from membrane passively is similar to small needles [10-11]. According to the importance of nanotubes in drug delivery and drug diagnosis sensors, this study examines adsorption of Lomustin with carbon nanotube. For this purpose, with computational method in computer, nanotube and drug were studied from different dimensions [12-13]. IUPAC and chemical structure of Lomustin are illustrated in Figure 1.

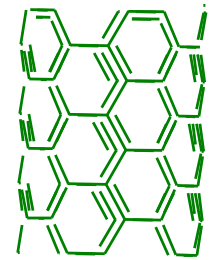

Nanotube

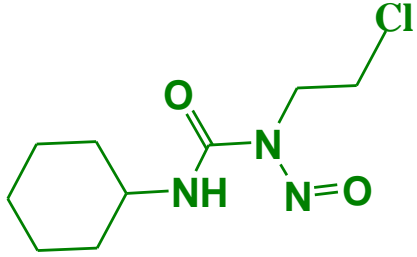

Lomustine

FIGURE 1 Chemical structure of Lomustin and carbon nanotube

\section{Computational methods}

First, the structure of Lomustin, carbon nanotube and their derived complexes was drawn and designed in three different modes using Gauss View 5.1[14] and Nanotube Modeler software [15], on which geometric optimization, IR, and molecular orbitals computations were performed, with 6-31G (d) basis set [16], and $B_{3} L Y P$ hybrid function [17]. This basis set was selected as in previous reports; the results of that match were in good experimental data [18]. All calculations were performed using Gaussian [19] and Spartan [20] softwares in the temperature range from 278.15 to $314.15 \mathrm{~K}$ over the temperatures range from $3^{\circ}$ to $3^{\circ}$. As a result, their optimum structure was obtained and, using the output of IR and natural bond orbital (NBO) [21] calculations, the absorption energy and other thermodynamic properties were determined using equation (2) [22]. The reactions in general are shown in the Equation 1:

$$
\text { Lomustin }+ \text { SWCNT } \rightarrow \text { Lomustin }- \text { SWCNT (1) }
$$

The energy of absorption between the Lomustin and carbon nanotubes is obtained according to Equation 2:

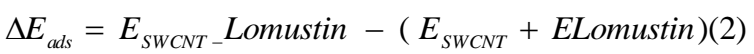

$\mathrm{E}_{\text {SWCNT-Lomustin, }} \mathrm{E}_{\text {Lomustin }}$ and $\mathrm{E}_{\text {SWCNT }}$ are the total molecular energy. $\Delta \mathrm{E}_{\text {ads }}$ is the energy absorbed by the Lomustin molecule on the surface of carbon nanotubes which is obtained at a level of 6-31G (d) / B B LYP. 


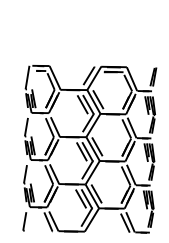

Nanotube<smiles>O=C(NC1CCCCC1)NN(CCCl)C(=O)O</smiles>

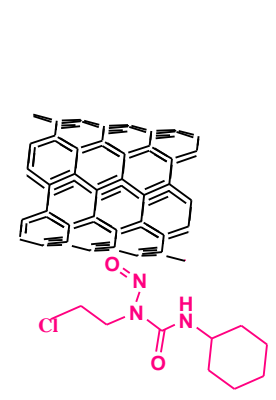

I-Isomer

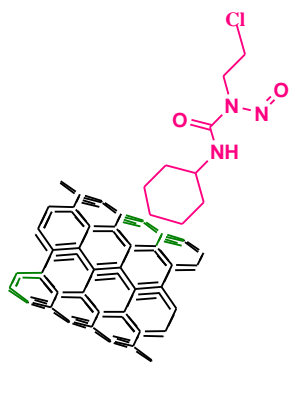

II-Isomer

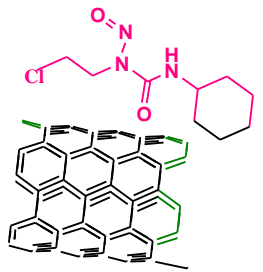

III-Isomer

FIGURE 2 Optimized structure of Lomustin and its derivatives with carbon nanotube

\section{Results and discussion}

\section{Study of structural properties}

As can be seen in Figure 2, three positions are closed to carbon nanotubes. In this regard, for easier understanding, each Lomustin derivate with carbon nanotubes is identified with an abbreviation and this naming method will be described in the future. Lomustin is closed to carbon nanotubes from three positions. Bonds between $\mathrm{C}-\mathrm{O}$ atoms and bonds between $\mathrm{N}=\mathrm{C}$ groups in Lomustin structure can have a key role in enhancing the effect of synthesized Lomustin. In other words, when these bonds are weak, Lomustin can enter the reaction more easily. For this reason, after geometrical optimization on all compounds, the length of
C-O bonds in pure Lomustin and the derivatives with carbon nanotubes were measured and the resulted values are presented in Tables 1 and 2. As data in Tables 1 and 2 indicate clearly, when $\mathrm{C}-\mathrm{O}$ and $\mathrm{C}-\mathrm{N}$ are closed to carbon nanotubes, the length of bonds in all three isomers is decreased and this means that these bonds have moved toward stable bonds and in this condition, looser electrons in the bonds of these derivatives can easily enter the process of effectiveness and reaction with compounds. Density is another parameter that has been examined in this study. As can be seen in the table, Lomustin density is decreased after adsorption by pure carbon nanotubes. This is related to a significant increase after carbon nanotubes adsorption with drug [23].

TABLE 1 Total energy values, the lowest observed frequency, bond distances, bond type, zero point energy, surface, mass, volume and density for Lomustin and its derivatives with carbon nanotube in the solvent phase

\begin{tabular}{ccccc}
\hline & Lomustin & I-Isomer & II-Isomer & III-Isomer \\
\hline $\mathrm{E}_{\text {ad }}($ water $)(\mathrm{kJ} / \mathrm{mol})$ & - & -1221.35 & -1264.31 & -1278.29 \\
lowest frequency $\left(\mathrm{cm}^{-1}\right)$ & 259644.00 & 13.90 & 12.35 & 22.81 \\
$\mathrm{C}-\mathrm{O}(\AA)$ & - & 1.41 & - & - \\
$\mathrm{C}-\mathrm{N}(\AA)$ & - & - & 1.46 & - \\
$\mathrm{C}-\mathrm{O}(\AA)$ & - & - & - & 1.43
\end{tabular}




$\begin{array}{ccccc}\text { zero point energy } & 801.62 & 1202.70 & 1191.17 & 1199.48 \\ (\mathrm{~kJ} / \mathrm{mol}) & 256.08 & 465.28 & 455.57 & 499.95 \\ \text { surface }\left(\AA^{2}\right) & 233.70 & 521.96 & 521.96 & 521.96 \\ \text { mass }(\mathrm{amu}) & 224.75 & 492.49 & 491.55 & 493.28 \\ \begin{array}{c}\text { volume }\left(\AA^{3}\right) \\ \text { density }=\text { mass } / \text { volume } \\ \left(\mathrm{amu} / \AA^{3}\right)\end{array} & 1.04 & 1.06 & 1.15 & 1.06\end{array}$

\section{Calculating and analyzing}

To obtain the values of the enthalpy adsorption carbon nanotube and Lomustin from the Equation (3). In this equation, $\Delta \mathrm{H}^{0}$ is the total energy variation in the process obtained by reduction in the total energy of the products of a reaction from the sum of the total energy of the raw material. $\Delta \mathrm{H}^{0}$ also represents the enthalpy sign for each of the reaction components [24].

$\Delta H_{a d}=E_{C W C N T}$ Lomustin $-\left(E_{\text {SWCNT }}+\right.$ ELomustin $)$ (3)

As the results show in Table 2, carbon nanotube reaction is done by exothermic Lomustin, and energy is transferred from the system to the environment, as the values of
$\Delta \mathrm{H}_{\mathrm{ad}}$ are obtained for all the derivatives are negative. However, this phenomenon cannot have an effect on the reaction run, because despite this increase, the enthalpy changes remains negative. Moreover, to examine the effect of temperature on carbon nanotube substituent process, all thermodynamic parameters were calculated at the temperature range from 278.15 to 314.15 Kelvin in the $3^{\circ}-3^{\circ}$ range and the values were reported. As is seen in Table 2, the temperature of the enthalpy changes gradually increases with increasing temperature. Thus, in the process of adsorption the desired compounds becomes warmer with increasing temperature [25-29].

TABLE 2 The values of enthalpy variations in the formation of substituent reaction of carbon nanotube and Lomustin in the water solvent phase at the temperature range from 278.15 to 314.15 Kelvin

\begin{tabular}{cccc}
\hline \multicolumn{4}{c}{$\boldsymbol{\Delta} \mathbf{H}_{\mathbf{a d}}(\mathbf{k J} / \mathbf{m o l})$} \\
\hline Temperature(K) & I-Isomer & II-Isomer & III-Isomer \\
\hline 278.15 & -1409.71 & -1201.71 & -1227.45 \\
281.15 & -1409.41 & -1201.40 & -1227.14 \\
284.15 & -1409.10 & -1201.08 & -1226.83 \\
287.15 & -1408.79 & -1200.75 & -1226.50 \\
290.15 & -1408.47 & -1200.41 & -1226.17 \\
293.15 & -1408.15 & -1200.08 & -1225.83 \\
296.15 & -1407.82 & -1199.74 & -1225.50 \\
299.15 & -1407.49 & -1199.39 & -1225.15 \\
302.15 & -1407.15 & -1199.04 & -1224.80 \\
305.15 & -1406.81 & -1198.67 & -1224.45 \\
308.15 & -1406.46 & -1198.31 & -1224.08 \\
311.15 & -1406.11 & -1197.93 & -1223.71 \\
314.15 & -1405.75 & -1197.55 & -1223.34 \\
\hline
\end{tabular}

Calculation and evaluation of Gibbs free energy changes and Lomustin derivatives with carbon nanotubes

Equation 4 was used to calculate the Gibbs free energy variation $\left(\Delta \mathrm{G}_{\mathrm{ad}}\right)$. Regarding this, $\Delta \mathrm{G}_{\mathrm{ad}}$ is the adsorption energy released by the Gibbs calculated by the software for each component of the reaction. The results, all presented in Table 3, show that in all the examined cases, carbon nanotubes are spontaneous on Lomustin. However, the amount of $\Delta \mathrm{G}_{\text {ad }}$ for isomer 1 is far worse than the Gibbs free 
energy variation for II-Isomer and III-Isomer. It is worth noting that by approaching the Lomustin to carbon nanotubes, the absorption process of both derivatives is significantly more spontaneous because the amount of $\Delta \mathrm{G}_{\mathrm{ad}}$ has experienced a sharp decline after the process. But, in general, given that the value of this parameter is substantially negative in all cases, it can be expected that the adsorption reaction of all compounds is possible empirically. Thus, the highest synthesis efficiency appears at room temperature or 298 Kelvin [30].

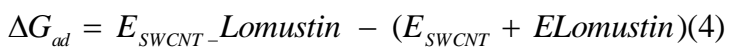

TABLE 3 Gibbs free energy change of carbon nanotube and Lomustin in the water solvent phase at the temperature range from 278.15 to 314.15 Kelvin

\begin{tabular}{cccc}
\hline \multicolumn{4}{c}{$\Delta G_{a d}(\mathrm{KJ} / \mathrm{mol})$} \\
\hline Temperature(K) & I-Isomer & II-Isomer & III-Isomer \\
\hline 278.15 & -1332.89 & -1126.29 & -1149.31 \\
281.15 & -1332.88 & -1126.31 & -1149.28 \\
284.15 & -1332.88 & -1126.31 & -1149.27 \\
287.15 & -1332.86 & -1126.31 & -1149.25 \\
290.15 & -1332.85 & -1126.31 & -1149.24 \\
293.15 & -1332.84 & -1126.31 & -1149.23 \\
296.15 & -1332.86 & -1126.33 & -1149.25 \\
299.15 & -1332.87 & -1126.35 & -1149.27 \\
302.15 & -1332.88 & -1126.38 & -1149.29 \\
305.15 & -1332.88 & -1126.42 & -1149.30 \\
308.15 & -1332.88 & -1126.46 & -1149.33 \\
311.15 & -1332.88 & -1126.48 & -1149.35 \\
314.15 & -1332.91 & -1126.52 & -1149.40 \\
\hline
\end{tabular}

TABLE 4 Specific heat capacity at different temperatures calculated at level B3lyp/ 6-31G (d) g *

\begin{tabular}{ccccc}
\hline \multicolumn{5}{c}{$\mathbf{C}_{\mathbf{v}} \mathbf{( J / m o l . K )}$} \\
\hline Temperature(K) & Lomustin & I-Isomer & II-Isomer & III-Isomer \\
\hline 278.15 & 202.850 & 389.9129 & 397.833 & 391.1211 \\
281.15 & 204.4875 & 394.0983 & 401.9891 & 395.3831 \\
284.15 & 206.1298 & 398.2838 & 406.1449 & 399.6435 \\
287.15 & 207.7769 & 402.469 & 410.300 & 403.9018 \\
290.15 & 209.4288 & 406.6533 & 414.4539 & 408.1575 \\
293.15 & 211.0854 & 410.8363 & 418.6063 & 412.4102 \\
296.15 & 212.7466 & 415.0174 & 422.7566 & 416.6593 \\
299.15 & 214.4123 & 419.1961 & 426.9044 & 420.9044 \\
302.15 & 216.0824 & 423.3719 & 431.0493 & 425.145 \\
305.15 & 217.7568 & 427.5445 & 435.1908 & 429.3806 \\
308.15 & 219.4354 & 431.7131 & 439.3285 & 433.6108 \\
311.15 & 221.118 & 435.8775 & 443.4619 & 437.8352 \\
314.15 & 222.8045 & 440.0371 & 447.5907 & 442.0532 \\
\hline
\end{tabular}

Specific heat capacity is the heat that should be received by a certain amount of substance to increase its temperature by $1 \mathrm{~K}$. Specific heat capacity of all compounds in the temperature range of 278.15 to $314.15 \mathrm{C}$ was calculated and reported in Table 4 . In this table, it can be observed that $\mathrm{C}_{\mathrm{v}}$ value in
Lomustin derivatives with nanotube is higher than Lomustin. Therefore, drug adsorption with nanotube leads to the reduced drug sensitivity to heat (Table 4). This issue, in the case of temperature sensitive drugs, is a positive point because in these drugs, with 
increased temperature, drug decomposition and inactivation are likely to occur [31].

\section{Calculating and verifying the thermodynamic constant of Lomustin and their derivatives with carbon nanotubes}

The constant thermodynamic adsorption of the synthesis of Lomustin derivatives with graphene was also calculated using Equation 5. In this equation, $\Delta \mathrm{G}_{\mathrm{ad}}$ is the same as the Gibbs free energy variation obtained at the previous stage, $\mathrm{R}$ is the ideal gas constant, and $\mathrm{T}$ is the temperature in Kelvin as the results presented in Table 5 clearly show.

$\Delta \mathrm{G}_{\mathrm{ad}}=-\mathrm{nRT} \ln \mathrm{K}$
In this study, the effect of adsorption of carbon nanotubes on structural properties of Lomustin was studied computationally.The thermodynamic parameters showed that the Lomustin drug reaction with carbon nanotubes is exothermic, spontaneous, oneway and non-equilibrium and this reaction has the highest efficiency at room temperature. Molecular orbit analysis also proved that carbon nanotubes derivatives have less conductivity, electrophilicity, and reactivity compared to pure Lomustin. As theoretical studies have shown, the graphene reaction with Lomustin is empirically possible. So the empirical investigation of the synthesis of these derivatives is highly recommended by experts in this field [32].

TABLE 5 The thermodynamic constant of Lomustin and their derivatives with carbon nanotube in the water solvent phaseat the temperature range from 278.15 to 314.15 Kelvin.

\begin{tabular}{cccc}
\hline & \multicolumn{2}{c}{$\mathbf{K}_{\text {th }}$} & \\
\hline Temperature(K) & I-Isomer & II-Isomer & III-Isomer \\
\hline 278.15 & $2.0693 \times 10^{+25}$ & $3.2951 \times 10^{+21}$ & $6.9219 \times 10^{+25}$ \\
281.15 & $4.4105 \times 10^{+27}$ & $1.8382 \times 10^{+29}$ & $3.4055 \times 10^{+23}$ \\
284.15 & $1.0700 \times 10^{+25}$ & $1.1351 \times 10^{+27}$ & $1.8817 \times 10^{+21}$ \\
287.15 & $2.9241 \times 10^{+22}$ & $7.7966 \times 10^{+24}$ & $1.1593 \times 10^{+29}$ \\
290.15 & $9.0525 \times 10^{+29}$ & $5.9311 \times 10^{+22}$ & $7.9447 \times 10^{+26}$ \\
293.15 & $3.1601 \times 10^{+27}$ & $4.9783 \times 10^{+20}$ & $6.0455 \times 10^{+24}$ \\
296.15 & $1.2500 \times 10^{+25}$ & $4.6508 \times 10^{+18}$ & $5.1474 \times 10^{+22}$ \\
299.15 & $5.5134 \times 10^{+22}$ & $4.7839 \times 10^{+16}$ & $4.807 \times 10^{+20}$ \\
302.15 & $2.7026 \times 10^{+23}$ & $5.3977 \times 10^{+14}$ & $4.9174 \times 10^{+198}$ \\
305.15 & $1.4676 \times 10^{+28}$ & $6.6622 \times 10^{+12}$ & $5.5138 \times 10^{+16}$ \\
308.15 & $8.8147 \times 10^{+25}$ & $8.9753 \times 10^{+10}$ & $6.7606 \times 10^{+14}$ \\
311.15 & $5.8513 \times 10^{+23}$ & $1.3052 \times 10^{+19}$ & $9.0385 \times 10^{+12}$ \\
314.15 & $4.3147 \times 10^{+21}$ & $2.0758 \times 10^{+17}$ & $1.3221 \times 10^{+11}$ \\
\hline
\end{tabular}

\section{Analysis of the results of calculations of} molecular orbitals

The most important frontier molecule orbital cell $\left(\mathrm{FMO}_{\mathrm{S}}\right)$, such as the highest occupied molecule orbital (HOMO) and the lowest unoccupied molecular orbital (LUMO) have a decisive role in the chemical stability of the molecule. The energy gap between HOMO and LUMO determines the reactivity, polarization, and hardness-softness of a molecule, which is represented by the Gap mark and used to calculate it using Equation 6. The energy gap is directly related to the molecular electrical conductivity. In fact, compounds that have small energy gaps can easily pass electrons from the barrier to the conductive strip, which means that materials that have less energy bands have more electrical conductivity than molecules with higher energy chats. The results presented in Table 6 clearly show that the energy gap after carbon nanotube connection has increased significantly. Indeed, the rate of conductivity of Lomustin has significantly decreased after carbon nanotube 
substituent. The next parameter examined is the chemical hardness ( $\eta$ ), whose value can be obtained using Equation 7. Chemical hardness is a good measure to estimate the reactivity of a new compound. This is because molecules that are structurally softer and have low chemical hardness can easily change their electron density. Thus, electronic transmissions essential for chemical reactions are better and easier to use in soft compounds. The data in the table shows that the reaction of Lomustin is reduced after the reaction with carbon nanotube since all the derivatives obtained from the carbon nanotubes subtraction reaction have a higher chemical hardness than the pure drug. The chemical potential $(\mu)$ used to obtain the rest of the parameters was calculated using Equation 8. Electrophilicity $(\omega)$ and the maximum load transmitted to the system $\left(\Delta \mathrm{N}_{\max }\right)$ are both suitable quantities, showing the tendency of a compound to absorb electrons. These two parameters were calculated using Equations 9 and 10 , respectively. When two molecules react with each another, one acts as an electrophile while another plays the role of a nucleophile and the compound whose electrophilicity and charge capacity are higher will tend to behave as an electron receptor. On the other hand, a molecule with low electrophilicity and capacity tends to accept the electron system. As shown in the Table 6, electrophilicity of Lomustin has been greatly reduced after carbon nanotube binding. Hence, one can conclude that the desire of Lomustin to absorb electrons has decreased. The dipolar state of the studied structures has also been studied. This parameter is a good criterion for evaluating the solubility of molecules in polar solvents. Molecules with higher dipole moments have better solubility in water and compounds with less bipolar moments will be weaker in polar solvents. As can be seen, the dipole moment of Lomustin decreases after carbon nanotube connection. Thus, carbon nanotube derivatives with Lomustin have less solubility in water compared to pure Lomustin [33]. Also Representation of the molecular structure of the Lomustin drug complexes with CNT (HOMO\& LUMO) in the three desired isomers Figure 3.

$$
\begin{aligned}
& H L G=E_{L}-E_{H} \\
& \eta=\left(E_{L}-E_{H}\right) / 2 \\
& \mu=\left(E_{L}+E_{H}\right) / 2 \\
& \omega=\mu^{2} / 2 \eta \\
& \Delta N_{\max }=-\mu / \eta
\end{aligned}
$$

TABLE 6 calculated HOMO and LUMO, band gap, chemical hardness $(\eta)$, chemical potential, electrophilicity $(\omega)$,the maximum amount of electronic charge index $\left(\Delta \mathrm{N}_{\max }\right)$ and dipole moment for the Lomustin and their derivatives with carbon nanotubes in the water solvent

\begin{tabular}{ccccccccc}
\hline & $\mathbf{E}_{\mathbf{H}}(\mathbf{e V})$ & $\mathbf{E}_{\mathbf{L}}(\mathbf{e V})$ & $\mathbf{H L G}(\mathbf{e V})$ & $\eta(\mathbf{e V})$ & $\boldsymbol{\mu}(\mathbf{e V})$ & $\boldsymbol{\omega}(\mathbf{e V})$ & $\boldsymbol{\Delta N}_{\max }(\mathbf{e V})$ & $\begin{array}{c}\text { Dipole } \\
\text { moments(Deby) }\end{array}$ \\
\hline Lomustin & -8.46 & 5.53 & 13.99 & 7.00 & -1.47 & -0.15 & 0.21 & 3.48 \\
I-Isomer & -6.31 & 1.59 & 7.90 & 3.95 & -2.36 & -3.31 & 0.60 & 3.47 \\
II-Isomer & -6.16 & 1.95 & 8.11 & 4.06 & -2.11 & -3.91 & 0.52 & 3.39 \\
$\begin{array}{c}\text { III- } \\
\text { Isomer }\end{array}$ & -6.57 & 1.35 & 7.92 & 3.96 & -2.61 & -3.00 & 0.66 & 2.60 \\
\hline
\end{tabular}




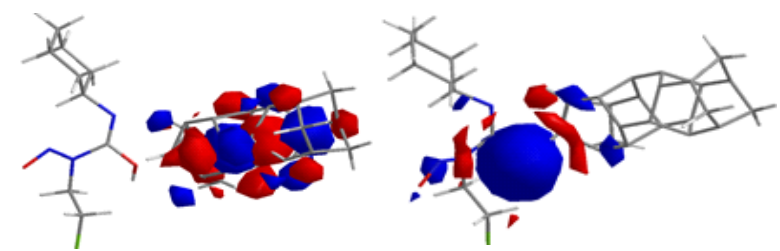

I-Isomer

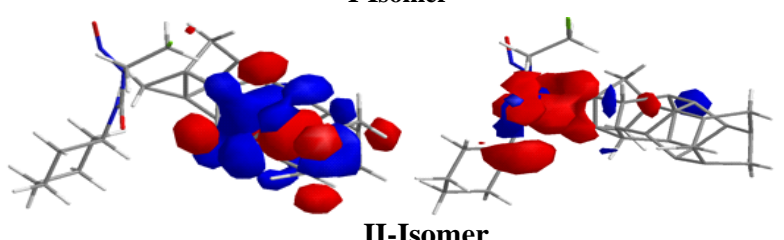

II-Isomer

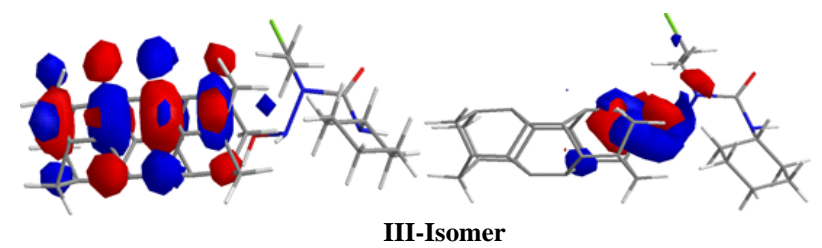

FIGURE 3 Representation of the molecular structure of the Lomustin drug complexes with CNT in the three desired isomers

\section{Conclusion}

The thermodynamic parameters showed that the Lomustin drug reaction with carbon nanotube is exothermic, spontaneous, and one sided and non-equilibrium and this reaction has the highest efficiency at room temperature. Molecular orbit analysis also proved that carbon nanotube derivatives have more conductivity, electrophilicity, and reactivity compared to pure Lomustin. As theoretical studies have exposed, the carbon nanotube reaction with Lomustin is empirically possible, so the experiential research of the adsorption of these derivatives is highly suggested by experts in this field.

\section{Acknowledgements}

The author appreciates Islamic Azad University of Tehran North Branch Research Council for the support of this project.

\section{Orcid:}

Roya Ahmadi: https://orcid.org/0000-00020002-7858

\section{References}

[1] A. Bahrami, S. Seidi, T. Baheri, M. Aghamohammadi, Superlattices. Micrstruct., 2013, 64, 265-273.

[2] M.D. Esrafili, Phys. Lett., 2017, 381, 20852091.

[3] A. Vinu, T. Mori, K. Ariga, Sci. Technol. Adv. Mater., 2006, 7, 753-771.

[4] A. Soltani, M.T. Baei, M. Mirarab, M. Sheikhi, E.T. Lemeshki, J. Phys. Chem. Solids., 2014, 75, 1099-1105.

[5] S.A. Siadati, M.S. Amini-Fazl, E. Babanezhad, Sens. Actuators. B. Chem., 2016, 237, 591-596.

[6] R. Rahimi, S. Kamalinahad, M. Solimannejad, Mater. Res. Express., 2018, 5, 117.

[7] P. Pakravan, S.A. Siadati, J. Mol. Graph. Model., 2017, 75, 80-84.

[8] M.T. Baei, Heteroatom. Chem., 2013, 24, 516-523.

[9] M. Soleymani, H.D. Khavidaki, Comput. Theor. Chem., 2017, 1112, 37-45.

[10] R. Ahmadi, M.R. Jalali Sarvestani, Phys. Chem. Res., 2018, 6, 639-655.

[11] M.R. Jalali Sarvestani, R. Ahmadi, Int. J. New. Chem., 2018, 4, 400-408. 
[12] M.R. Jalali Sarvestani, R. Ahmadi, Int. J. New. Chem., 2018, 5, 409-418.

[13] R. Ahmadi, M.R. Jalali Sarvestani, Int. J. Bio-Inorg. Hybrid. Nanomater., 2017, 6, 239244.

[14] R. Ahmadi, Int. J. Nano. Dimens., 2017, 8, 250-256.

[15] A. Mohasseb, Int. J. New Chem., 2019, 4, 215-223.

[16] R. Ghiasi, F. A. K. Kanani, Asian J. Nanosci. Mater., 2018, 1, 234-243.

[17] R. Ahmadi, M. Pirahan-Foroush, Ann Mil Health Sci Res., 2016, 12, 86-90.

[18] Ö. Alver, M. Bilge, N. Atar, C. Parlak, J Mol Liq., 2017, 231, 202-205.

[19] R. Ahmadi, T. Boroushaki, M. Ezzati, Orient J Chem., 2014, 28, 773-779.

[20] S. Bashiri, E. Vessally, Bekhradnia, A. Hosseinian A.L. Edjlali, Vacuum., 2016, 136, 156-162.

[21] R. Ahmadi, M.R. Jalali Sarvestani, Sadeghi B., Int. J. Nano Dimens., 2018, 9, 325-335

[22] R. Ahmadi, E. S. Mirkamali, J. Phys Theor. Chem. IAU, Iran., 2016, 13, 297-302.

[23] Y. Gökpek, M. Bilge, D. Bilge, Ö.Alver, C. Parlak, J Mol Liq., 2016, 238, 225-228.

[24] R. Ahmadi, T. Boroushaki, M. Ezzati, Int. J. Nano Dimens., 2015, 6, 19-22.
[25] R. Ahmadi, M. Pirahan-Foroush, Ann. Mil Health Sci Res., 2015, 12, 39-43.

[26] R. Ahmadi, Int. J. Nano Dimens., 2017, 83, 250-256.

[27] J.S. Rodman, D.J. Deutsch, S.I. Gutman, A.M. J. Medi., 1976, 60, 941-948.

[28] R. Ahmadi, J Phys Theor Chem IAU, Iran., 2012, 9, 185-190.

[29] R. Ahmadi, S. Pourkarim, Int. J. Bio-Inorg Hybd Nanomat., 2015, 4, 249-254.

[30] R. Ahmadi, R. Rezaei asl, Int. J. New Chem., 2015, 4, 189-198.

[31] R. Ahmadi, M. Salmaniha, Int. J. New Chem., 2015, 4, 152-160.

[32] F. Alzahar, Int. J. New Chem., 2017, 4, 111117

[33] M. Noei, A. Moalla, Int. J. New Chem., 2014, 3, 99-107.

How to cite this article: Maryam Afshar, Roya Ranjineh khojasteh, Roya Ahmadi*. Adsorption of lomustin anticancer drug on the surface of carbon nanotube: A theoretical study. Eurasian Chemical Communications, 2020, 2(5), 595-603. Link:

http://www.echemcom.com/article_1018 09.html 